\title{
Tuberculose em necropsias realizadas no Serviço de Anatomia Patológica da Faculdade de Medicina de Botucatu
}

\author{
Tuberculosis in necropsies performed at the Department of Pathology of Botucatu Medical School
}

Andrei Fernandes Joaquim'; Luana Carandina²; Julio Defaveri ${ }^{3}$

\begin{abstract}
unitermos resumo
Tuberculose

Entre 6.316 necropsias realizadas, no período de 1969 a 2000, no Departamento de Patologia da Fa-

Necropsias

culdade de Medicina de Botucatu da Universidade Estadual Paulista (FMB/UNESP), foram encontradas 240 necropsias com diagnóstico de tuberculose, sendo 117 registradas como doença principal (grupo

Patologia tuberculose/doença principal) e 123, como doença associada (grupo tuberculose/doença associada). No grupo tuberculose/doença principal, 100\% apresentavam tuberculose ativa, com 80 ocorrências em homens e 37 em mulheres e média de idade de 47,7 anos. Caquexia $(37,2 \%)$ e cor pulmonale $(23,7 \%)$ foram as co-morbidades mais prevalentes nesse grupo. Os pulmões estavam comprometidos em $95,7 \%$ dos casos, seguido pelos linfonodos $(38,9 \%)$ e pleuras $(27,1 \%)$. Os padrões morfológicos das lesões pulmonares foram: 1 . miliar, 58,4\%; 2. cavernas, $56,6 \%$; 3. fibrose, $41,5 \%$; 4 . bronquiectasias, $26,5 \%$; 5. enfisema, 19,4\%; e 6. cistos, 1,7\%. Cavernas foram observadas em diferentes áreas, com discreto predomínio no pulmão direito, sendo a localização apical preferencial (100\%) e quase sempre bilaterais (94\%). Lesões miliares, bronquiectasias e fibrose estavam distribuídas, de forma difusa e aleatória, nos pulmões. No grupo tuberculose/doença associada também houve predomínio de homens (70,8\%). Nesses casos, a AIDS e o alcoolismo crônico foram as doenças principais mais freqüentes, representando $22 \%$ e $16,3 \%$, respectivamente. Os pulmões estavam acometidos em $85,2 \%$ dessas necropsias (100\% nos casos de AIDS), seguidos pelos linfonodos (31,9\%) e baço $(28,6 \%)$. Nossos dados corroboram a importância da necropsia no estudo e no conhecimento da tuberculose, fornecendo subsídios para uma melhor abordagem clínica e epidemiológica dessa doença em nossa região.
\end{abstract}

Among 6,316 necropsies performed in the period 1969-2000 at the Department of Pathology of Botucatu Medical School UNESP, 240 were of tuberculosis. Among them, 117 were registered as the principal disease (tuberculosis/principal disease group) and 123, as associated to another disease (tuberculosis/associated disease group). In the tuberculosis-principal disease group, 100\% had active tuberculosis, 80 patients were male and 37 were female, with mean age of 47.7 years. Cachexia (37.2\%) and cor pulmonale (23.7\%) were the most prevalent comorbities in this group. The lungs were affected in $95.7 \%$ of the cases, followed by lymph nodes (38.9\%) and pleura (27.1\%). The morphology and frequency of pulmonary lesions were: 1. miliar: 58.4\%; 2. caverns, 56.6\%; 3. fibrosis, $41.5 \%$; 4. bronchiectasias, $26.5 \%$; 5. emphysema, $19.4 \%$ and 6. cysts, $1.7 \%$. Caverns were observed in different areas of both lungs, with discreet predominance in the right lung, were located mainly in the apices (100\%), and the great majority (94\%) was bilateral. The others lesions were distributed randomly in both lungs. In the tuberculosis/associated disease group, $70.8 \%$ was male, and the two most frequent principal diseases were AIDS (22.1\%) and alcoholism (16.3\%). Also, the lung was the most affected organ (85.2\%), followed by lymph nodes (31.9\%) and spleen (28.6\%). In 100\% of AIDS there were pulmonary lesions. This study in necropsies reinforces epidemiological data that tuberculosis is an important disease, and, also, constitutes an invaluable tool to gather information for a better understanding of the pathology and the epidemiology of tuberculosis in our region.

1. Graduando em Medicina; bolsista do Programa Institucional de Bolsas de Iniciação Científica (PIBIC).

2. Professora-doutora do Departamento de Saúde Pública da Faculdade de Medicina de Botucatu da Universidade Estadual Paulista (FMB/UNESP).

3. Professor-adjunto do Departamento de Patologia da FMB/UNESP.

Trabalho realizado no Departamento de Patologia da FMB/UNESP. 


\section{Introdução}

Dados da Organização Mundial da Saúde (OMS) ${ }^{(23)}$ mostram o ressurgimento de inúmeras doenças endêmicas, entre elas a tuberculose (TB) a doença infecciosa que mais mata jovens e adultos no mundo.

No Brasil, estima-se haver 50 milhões de infectados e 129 mil casos novos de TB anualmente, sendo que desses apenas 90 mil casos são notificados. O país ocupa a sexta colocação no mundo em número de casos de TB, tendo sido oficialmente registrados, em 1996, 5.928 óbitos por $\mathrm{TB}^{(15)}$.

Embora o número de casos de TB notificados não tenha variado muito entre 1985 e 1998, sabe-se que esse número não condiz com a realidade, pois o sistema de notificação é falho, uma vez que há dificuldades em se diagnosticar a doença ou mesmo pelo fato de os doentes não procurarem os postos de saúde. Ainda agrava esse quadro o fato de que nos países em desenvolvimento e subdesenvolvidos, embora a incidência da TB fosse sempre elevada, foi pouco enfatizada no ensino das diversas áreas de saúde, pois a maioria das escolas médicas orientava seu ensino baseando-se na literatura publicada no meio científico internacional, a qual reflete predominantemente a realidade social dos países desenvolvidos, onde a incidência da tuberculose é baixa há muitas décadas ${ }^{(22)}$.

Para melhor caracterizar e entender os diferentes aspectos anatomopatológicos envolvidos na TB, a realização e a análise de necropsias têm papel importante. Contudo, apesar de importantes, esses estudos são pouco relatados ${ }^{(1-10,14,16,17-21)}$, quando comparados com o número de estudos clínicos e epidemiológicos utilizados de tempos em tempos para o estabelecimento de diretrizes e normas de conduta para os profissionais da saúde, frente a essa importante doença ${ }^{(11)}$. Na literatura nacional há poucos trabalhos em necropsias e, nesses estudos, os relatos são por órgãos ou sistema, não sendo analisado seus aspectos anatomopatológicos no organismo como um todo ${ }^{(2,8-10)}$.

Do exposto, e considerando a importância da TB em nosso meio, este trabalho teve como objetivo rever as necropsias de TB realizadas no Departamento de Patologia da Faculdade de Medicina de Botucatu (FMB), a fim de conhecer nossa realidade regional e obter subsídios anatomopatológicos para a melhor compreensão da patogenia dessa doença.

\section{Material e métodos}

A área geográfica do presente estudo é a região de Botucatu, localizada no Centro-Oeste do Estado de São
Paulo. Essa região (DIR XI) abrange 31 municípios, com uma população estimada em 572.101 habitantes para 2006. Todos os municípios seguem orientações do Programa Nacional de Controle da Tuberculose e têm como referência especializada o Hospital das Clínicas da Faculdade de Medicina de Botucatu da Universidade Estadual Paulista (FMB/UNESP) ${ }^{(22)}$.

Foram analisados os relatórios das necropsias com diagnóstico de TB registradas nos arquivos do Departamento de Patologia da Faculdade de Medicina, entre 1969 e 2000. Todas as necropsias eram completas, com exames macro e microscópico de todos os órgãos. Os casos foram divididos nos grupos tuberculose/doença principal (TB/DP) e tuberculose/doença associada (TB/DA).

Dos relatórios foram extraídas as seguintes informações:

- dados clínicos: ano da necropsia, procedência, sexo, cor, idade e profissão do paciente, ausência/presença de tratamento e se houve diagnóstico clínico;

- dados anatomopatológicos, macro e microscópicos: órgãos e cadeias linfáticas acometidas (pulmões: tipo e local de acometimento das lesões);

- doenças associadas (grupo TB/DP) e doença principal (grupo TB/DA).

Foram consideradas como TB ativa os casos com exame macroscópico compatível com o diagnóstico (pneumonia caseosa, nódulos caseosos ou fibrocaseosos e miliares, e cavernas), e microscópico com granulomas epitelióides (com/sem células gigantes e com/sem necrose caseosa), além da presença do bacilo detectado pela coloração de Ziehl-Neelsen.

\section{Resultados}

Das 6.316 necropsias realizadas entre 1969 e 2000, a TB foi detectada em 3,8\% (240 casos). Dessas, 117 necropsias foram caracterizadas como TB/DP e 123 como TB/DA. Quando distribuídas nos períodos de 1969 a 1985 e 1986 a 2000, observou-se aumento do número de casos de TB/ DA no período de 1986 a 2000 (78 casos), em relação ao período de 1969 a 1985 (44 casos). No grupo TB/DP, no segundo período, houve pequena diminuição do número de casos (de 68 para 50). Houve predomínio de homens tanto no grupo TB/DP $(67,7 \%)$ (Tabela 1) quanto no TB/DA (70,8\%). Na maioria dos casos do grupo TB/DP, a TB ocorreu após os 30 anos, com média de idade de 47,7 
anos. Apenas $9,4 \%$ dos pacientes tinham menos de 20 anos. Nesse grupo, $72 \%$ dos pacientes eram brancos, seguidos de pardos (14,4\%), negros (9,3\%), e não houve referência à tez em $4,3 \%$ desse grupo. Na maioria dos casos $(47,5 \%)$ não havia registro da profissão, mas quando registrado, a maioria $(23,7 \%)$ era composta de lavradores. A doença estava em atividade em $100 \%$ dos casos de TB/DP, contra $72,5 \%$ dos casos do grupo TB/DA.
A freqüência dos principais órgãos acometidos nos grupos TB/DP e TB/DA está expressa na Tabela 2. Em ambas as séries houve predomínio de lesões pulmonares e de linfonodos regionais. No grupo TB/DP o comprometimento pulmonar foi observado em 113 casos (95,7\%), sendo que em $22(19,4 \%)$ exclusivamente nesse órgão. Em cinco casos (4,3\%) a TB era exclusivamente extrapulmonar. O comprometimento pleural foi maior no grupo TB/DP

Tabela 1

\section{Distribuição da freqüência segundo sexo e faixa etária de 117 necropsias do grupo}

\begin{tabular}{cccc|}
\hline Faixa etária (em anos) & Masculino & Feminino & Total \\
\hline$<1$ & 0 & 1 & 1 \\
$1-4$ & 1 & 4 & 5 \\
$5-14$ & 0 & 3 & 3 \\
$15-19$ & 0 & 2 & 11 \\
$20-29$ & 7 & 4 & 14 \\
$30-39$ & 8 & 6 & 26 \\
$40-49$ & 22 & 4 & 22 \\
$50-59$ & 18 & 4 & 33 \\
$>60$ & 23 & 10 & 117 \\
Total & 79 & 38 & \multicolumn{2}{c}{} \\
\hline
\end{tabular}

Tabela 2

\begin{tabular}{ll}
\hline Órgão & Núm \\
Pulmões & 1 \\
Linfonodos & \\
Pleura & 32 \\
Intestinos & 27 \\
Baço & 26 \\
Fígado & \\
Rins & \\
Meninges & \\
Peritônio & \\
Encéfalo & \\
Supra-renal & \\
Medula óssea & \\
Próstata & \\
Pâncreas & \\
\hline
\end{tabular}

Comparação entre a freqüência de acometimento por órgãos em 117 necropsias do grupo tuberculose/doença principal (TB/DP) e em 123 necropsias do grupo tuberculose/doença associada (TB/DA)

Grupo IB/DP

Grupo TB/DA

\begin{tabular}{cccc} 
Túmero & $\%$ & Número & $\%$ \\
113 & 95,7 & 104 & 85,2 \\
46 & 38,9 & 39 & 31,9 \\
32 & 27,1 & 8 & 6,5 \\
27 & 22,8 & 14 & 11,4 \\
26 & 22 & 35 & 28,6 \\
26 & 22 & 28 & 22,9 \\
22 & 18,6 & 22 & 18 \\
12 & 10,1 & 3 & 2,4 \\
10 & 8,4 & 9 & 7,3 \\
8 & 6,8 & 1 & 0,8 \\
6 & 5 & 8 & 6,5 \\
4 & 3,3 & 16 & 13,1 \\
4 & 3,3 & 3 & 2,4 \\
3 & 2,5 & 7 & 5,7 \\
\hline
\end{tabular}


$(27,1 \%)$ do que no TB/DA (6,5\%). O mesmo foi observado nas meninges, com 12 casos $(10,1 \%)$ e $3(2,4 \%)$, respectivamente. Da mesma forma, o acometimento intestinal foi maior no grupo TB/DP (22,8\%) do que no TB/DA (11,4\%). No grupo TB/DA houve acometimento importante de medula óssea $(13,1 \%)$, contra apenas 4 $(3,3 \%)$ do TB/DP.

Os padrões macroscópicos das lesões pulmonares nos 113 casos do grupo TB/DP tiveram a seguinte distribuição: $58,4 \%$ das lesões eram do tipo miliar e em $56,6 \%$ havia cavernas. Essas foram observadas em diferentes áreas, com discreto predomínio no pulmão direito, sendo a localização apical preferencial (100\%), e quase sempre bilaterais (94\%) (Tabela 3). Fibroses (41,5\%), bronquiectasias $(26,5 \%)$ e enfisemas $(19,4 \%)$ eram difusos e irregularmente distribuídos no parênquima pulmonar. Cistos raramente foram referidos, apenas $1 \%$.
Os linfonodos acometidos foram, em sua maioria, do hilo pulmonar, mediastinais e peritraqueais $(31,6 \%)$, seguidos das cadeias mesentéricas $(11,1 \%)$ e, com menor freqüência, periaórticas (5,1\%) e cervicais (3,5\%) (Tabela 4).

No grupo TB/DA, a doença principal mais freqüente foi a AIDS (22\%), seguida pelo etilismo crônico (16,3\%) (Tabela 5). Nos 27 casos de AIDS, a TB ativa foi detectada em 25 casos (92,6\%), e em apenas dois casos $(7,4 \%)$ foi referido tratamento.

Os órgãos mais acometidos nesse grupo de pacientes foram: pulmão (100\%); baço (76\%); fígado (76\%); linfonodos (68\%); rins (56\%); intestinos (44\%); medula óssea (40\%); peritônio (32\%); supra-renal (28\%); pâncreas (24\%); coração (24\%); e encéfalo (8\%).

Foram avaliados 43 prontuários dos 118 casos de TB/DP. Desses, 29 casos (67,5\%) tiveram diagnóstico de TB em vida, contra 14 (32,5\%) com diagnóstico da doença somente em necropsia.
Tabela 3

\begin{tabular}{rcccc} 
Tabela 3 & grupo tuberculose/doença principal (TB/DP)* & & \\
\hline Local & Miliar & Cavernas & Fibrose & Bronquiectasia \\
APD & 41 & 33 & 26 & 13 \\
MPD & 30 & 11 & 6 & 13 \\
IPD & 33 & 8 & 8 & 12 \\
APE & 41 & 31 & 20 & 10 \\
IPE & 36 & 13 & 6 & 13 \\
Total & $66 / 113(58,4 \%)$ & $64 / 113(56,6 \%)$ & $47 / 113(41,5 \%)$ & $30 / 113(26,5 \%)$ \\
\hline
\end{tabular}

APD: ápice do pulmão direito; MPD: lobo médio do pulmão direito; IPD: lobo inferior direito; APE: ápice do pulmão esquerdo; IPE: Iobo inferior esquerdo.

* Em quatro casos não havia registro de comprometimento pulmonar. Tabela 4 necropsias do grupo tuberculose/doença principal (TB/DP)

\begin{tabular}{ccc}
\hline Cadeias linfonodais & Número & $\%$ \\
\hline Hilo pulmonar & 17 & 14,5 \\
Peritraqueais & 5 & 4,3 \\
Mediastinais & 15 & 12,8 \\
Mesentéricos & 13 & 11,1 \\
Periaórticos & 6 & 5,1 \\
Cervicais & 4 & 3,5 \\
Axilares & 3 & 2,6 \\
Inguinais & 2 & 1,7 \\
Pericárdicos & 1 & 0,9 \\
\hline
\end{tabular}




\begin{tabular}{|c|c|c|}
\hline \multicolumn{3}{|c|}{$\begin{array}{l}\text { "Doenças principais" mais freqüentes em } 123 \text { necropsias do grupo tuberculose/doença } \\
\text { associada (TB/DA) }\end{array}$} \\
\hline Doença principal & Número & $\%$ \\
\hline AIDS & 27 & 22 \\
\hline Etilismo crônico & 20 & 16,3 \\
\hline Diabetes mellitus & 6 & 4,9 \\
\hline Doença de Chagas & 6 & 4,9 \\
\hline Hipertensão arterial & 4 & 3,2 \\
\hline Tabagismo crônico & 3 & 2,4 \\
\hline
\end{tabular}

\section{Discussão}

Das 6.316 necropsias realizadas nos 31 anos do Serviço de Anatomia Patológica da FMB, a TB representou 3,8\%. Dessas, a TB era a doença principal (TB/DP) em 49\%, representando TB ativa clinicamente, e, em $51 \%$, a TB era doença associada (TB/DA), sendo freqüentemente descoberta na necropsia.

Essa alta porcentagem de TB nas necropsias resulta da incidência média anual de 40 casos novos de TB por 100 mil habitantes observada na região de Botucatu ${ }^{(22)}$, bem como do fato de o Hospital das Clínicas da FMB ser o centro de referência para TB na região.

Houve predomínio de homens em ambos os grupos, TB/DP $(67,7 \%)$ e TB/DA (70,8\%). Na literatura, nos casos de TB ativa é referida maior incidência em homens, em proporções homem/mulher variáveis de 1,5 a 2,5/1(5, 6, 12).

A média de idade nos casos de TB/DP foi de 47,7 anos, revelando o predomínio de pacientes com mais de 40 anos e indicando que a tuberculose é secundária na maioria dos casos, uma vez que a maior parte da população infecta-se nos primeiros anos de vida ${ }^{(3,12)}$. Em um número significativo de laudos não havia registro da profissão. A ocorrência em 28 casos $(23,7 \%)$ de TB em lavradores foi inesperada, tendo em vista o caráter urbano da doença ${ }^{(22)}$. É possível que isso se deva às condições socioeconômicas e ao confinamento que essa população de lavradores é submetida nos períodos da colheita.

Os achados clínicos e anatomopatológicos no grupo TB/DP foram bastante variados, predominando manifestações sistêmicas graves relacionadas à TB, como desnutrição/caquexia, cor pulmonale e broncopneumonia, que são manifestações clínicas resultantes do caráter crônico e progressivo da TB. Nesse grupo foi alta a incidência de tromboembolismo pulmonar (TEP) $(13,5 \%)$ e de esofagites $(17,5 \%)$. Os fatores de risco bem estabelecidos para explicar a frequiência relativamente alta de TEP são o tabagismo e o possível tempo prolongado em que o paciente ficou acamado. Para as esofagites, pode estar associado o alcoolismo, hábito comum nos pacientes com TB.

Em ambos os grupos, como esperado, houve predomínio do comprometimento pulmonar. $\mathrm{O}$ acometimento pulmonar é o mais freqüente em todos estudos de necrop$\operatorname{sias}^{(2,6,12,17,19-21)}$, variando de $80 \%$ a $100 \%$, bem como o comprometimento de outros órgãos do sistema monocíticomacrofágico, como linfonodos, baço e fígado.

Dos 113 casos de TB/DP com acometimento pulmonar, $58,4 \%$ das lesões eram do tipo miliar, semelhante ao relatado na literatura ${ }^{(1-3,5,5,12,14,16,17,19-21)}$. Ao se considerar as circulações linfática e sanguínea, o pulmão torna-se, de forma recorrente, sua própria fonte de infecção, pois de lesões tuberculosas ativas os bacilos são drenados por via linfática, cuja circulação desemboca no sistema venoso ${ }^{(18)}$ e, então, prossegue para o átrio direito, novamente retornando e infectando o pulmão via artéria pulmonar. Além dessa importante via de disseminação, embora com menor probabilidade, os bacilos que ultrapassassem a barreira capilar pulmonar seriam drenados pelas veias pulmonares e cairiam na aorta, podendo daí voltar aos pulmões via artérias brônquicas.

Essas vias de disseminação do bacilo explicam a alta freqüência de lesões miliares pulmonares, quase sempre presentes nas afecções pulmonares crônicas (infecciosas ou não). Além dessas vias vasculares, a TB também pode se disseminar nos pulmões por via canalicular e via poros de Khon ${ }^{(3,12)}$. 
As mais freqüentes após as lesões miliares foram as lesões apicais, representadas principalmente por cavernas e fibroses. Nesta casuística as cavernas representaram 56,6\% das lesões, sendo observadas em todos os lobos. Porém, as cavernas estavam localizadas sempre nos ápices pulmonares e eram bilaterais em $94 \%$ dos casos. Esse achado difere da literatura, que relata, na grande maioria dos casos, a presença de cavernas em apenas um dos ápices pulmonares ${ }^{3,19)}$. Não há consenso na literatura sobre a razão da localização apical na TB.

Smith et al. (apud Balasubramanian et al..$^{[3]}$ ) coletaram pelo menos 16 teorias sobre como os bacilos ali chegam, se fixam e proliferam. A hipótese mais aceita é a de que as lesões apicais sejam mais abundantes pela maior disponibilidade de oxigênio nessa região, a qual possui melhor relação ventilação/perfusão, possibilitando assim a proliferação do bacilo, a confluência de lesões granulomatosas, o estabelecimento da necrose caseosa e a conseqüente formação de cavernas ${ }^{(3)}$. Assim, a alta freqüência de lesões apicais na $T B$, principalmente se representadas por cavernas, torna-as um forte indicador radiológico para o diagnóstico da $T B$, sugerindo ao clínico tratar o caso como TB, mesmo quando os testes para confirmação bacilar ou de outro agente etiológico forem negativos.

Outras lesões sem localização preferencial nos pulmões também foram freqüentes, como as bronquiectasias, que podem ser explicadas pelo freqüente comprometimento tuberculoso linfático do interstício broncovascular, causando o enfraquecimento da parede bronquial, associado a trações das vias aéreas, exercidas pela retração cicatricial de lesões que ocorrem no parênquima peribrônquico.

No grupo TB/DP chamou atenção o acometimento intestinal (22,8\%), sendo esse sítio o quarto mais atingido, o que contrasta com o relatado por Martinez et al.(16) $\mathrm{e}$ Rowinska-Zakrzewska et al. ${ }^{(19)}$, que observaram, respectivamente, $3,7 \%$ e $2,7 \%$ de comprometimento desse órgão. Várias hipóteses podem ser levantadas para explicar esses achados, como registro incompleto das alterações nos laudos das necropsias, metodologias divergentes nas realizações das necropsias, infecção intestinal pelo Mycobacterium bovis devido à ingestão de leite in natura e de seus produtos derivados, e ainda por diferenças na virulência entre as cepas de bacilos nas regiões referentes aos estudos, acarretando padrões de disseminação diversos do bacilo após a infecção pulmonar(13). Como conseqüência do acometimento do trato digestório, $11,1 \%$ dos casos apresentavam acometimento dos linfonodos mesentéricos, o que reforça a importância da via linfática na disseminação da doença.

No grupo TB/DA, possivelmente pelas doenças imunossupressoras de base, permitindo maior proliferação e disseminação bacilar, houve acometimento importante de medula óssea em 16 casos $(13,1 \%)$, contra apenas quatro casos (3,3\%) no grupo TB/DP.

Em 86,2\% das necropsias, a TB estava em atividade. A porcentagem de casos de TB ativa no total de necropsias encontradas em nosso serviço foi de $3,2 \%$, sendo superior a de outros trabalhos na literatura com 1,97\%(5) e 2,8\%(6), respectivamente. Essas diferenças epidemiológicas podem ser devido a diferentes incidências regionais de TB, à eficácia dos sistemas de saúde locais, à existência de serviços de referências, ao aparecimento de multirresistência aos antibióticos e/ou ainda por uma possível diferença regional na virulência do bacilo.

Houve aumento do número de casos de TB/DA no período de 1986 a 2000, em relação ao primeiro período de 1969 a 1985. Observamos também um aumento do número total de casos de TB no segundo período. Essas mudanças podem ser explicadas pelo impacto epidemiológico da AIDS na tuberculose, descrito inicialmente a partir da década de $1980^{(23)}$. A AIDS foi a doença de base mais prevalente no grupo TB/DA, com 27 casos (22\%). Salientase que só há relatos de diagnósticos de AIDS nos laudos de necropsia a partir de 1989, o que aumenta ainda mais sua prevalência relativa sobre os casos de TB/DA no período de 1989 a 2000.

A segunda doença principal mais freqüente nas necropsias do grupo TB/DA foi o etilismo crônico (16,3\%), destacando-se ainda a doença de Chagas, com seis casos $(4,9 \%)$.

Neste trabalho, a freqüência de acometimento pulmonar pela TB nos pacientes com AIDS diferiu do observado na literatura. Todos os nossos casos apresentavam acometimento pulmonar, comparado a apenas 56\% relatado por Abdel-Dayem et al. ${ }^{(1)}$. Possíveis diferenças nas cepas virais do HIV, na epidemiologia local do HIV, e ainda diferenças qualitativas na amostragem macro/microscópicas dos pulmões, devem ser consideradas para explicar tais divergências. Acometimento pulmonar exclusivo ocorreu 
em cinco casos (20\%) nos pacientes com AIDS, freqüência similar à do grupo TB/DP (19,4\%).Quanto ao momento do diagnóstico da TB, a freqüência de diagnósticos da TB em vida em nosso serviço $(67,4 \%)$ foi semelhante ao relatado na literatura, com variação de $40 \%$ a $70 \%$ dos $\operatorname{casos}^{117,19}$ ${ }^{20)}$. Na maioria dos casos de TB/DA o diagnóstico foi achado de necropsia, possivelmente devido à dificuldade de se identificar clinicamente a TB.

A tuberculose é uma doença complexa e, apesar de inúmeros estudos clínicos e experimentais, muitos dos seus aspectos patogenéticos continuam desconhecidos. Nossos dados corroboram a importância da necropsia para o estudo da tuberculose, fornecendo subsídios para um melhor entendimento de sua patogenia, e para uma melhor abordagem clínica e epidemiológica dessa importante doença.

\section{Agradecimentos}

À Dra. Márcia Guimarães da Silva, docente do Departamento de Patologia da Faculdade de Medicina de Botucatu da Universidade Estadual Paulista (FMB/UNESP), pelas sugestões e revisão do texto.

\section{Referências}

I. ABDEL-DAYEM, H.M. et al. Sites of tuberculous involvement in patients with AIDS. Autopsy findings and evaluation of gallium imaging. Clin Nucl Med, v. 22 n. 5, p. 310-4 1997.

2. ALMEIDA, M.C. et al. Anatomo-clinical diagnosis correlation: retrospective assessment of the clinical diagnosis in necropsies. Rev Saúde Pública, v. 23, p. 285-9|, 1989.

3. BALASUBRAMANIAN, $\vee$. et al. Pathogenesis of tuberculosis: pathway to apical localization. Review Tuber Lung Dis, v. 75 p. 68-178, 1994

4. BAKHSHI, S.S.; HAWKER, J.; ALI, S. Tuberculosis mortality in notified cases from 1989-1995 in Birmingham. Public Health, v. I12, p. 165-8, 1998

5. BOBROWITZ, I.D.Active tuberculosis undiagnosed until autopsy. Am J Med, v. 72, p. 650-8, 1982.

6. CHASTONAY, P.; GARDIOL, D. Extensive active tuberculosis at autopsy: retrospective study of a collection of adult autopsies (1961-1985). Schweiz-Med-Wochenschr, v. II7, n. 24, p. 925-7, 1987.

7. COLE, C.H. et al.TB and AIDS: Florida. MMWR, v. 35, p. 587-90, 1986.

8. COUTINHO, L.M.; TARRAGO, R.P. Meningo-encephalic tuberculosis in childhood: anatomo pathological study of 10 cases. Arq Neuropsiquiatr, v. 34, p. 127-34, 1976.

9. FERNANDES, V.S. et al. Incidence of adrenalitis in necropsy material. Rev Hosp Clín Fac Med São Paulo, v. 46, p. 21 9-22, |99|.

10. GUTIERREZ, E.B. et al. Autopsy-proven determinants of death in HIV-infected patients treated for pulmonary tuberculosis in São Paulo, Brazil. Pathology Research and Practice, v. 198 p. 339-46, 2002

| ।. || DIRETRIZES BRASILEIRAS PARA A TUBERCULOSE - 2004. J Bras Pneumol, v. 30, supl. I, 2004.

12. KAYNE, G.G.; PAGEL, W.; SHAUGHNESSY, L.O. Pulmonary tuberculosis: pathology, diagnosis, management and prevention. New York: Oxford Medical Publication, 1948.

13. LÓPEZ, B. et al.A marked difference in pathogenesis and immune response induced by different Mycobacterium tuberculosis genotypes. Clin Exp Immunol, v. I33, p. 30-7, 2003.

14. MACGEE,W.The frequency of unsuspected tuberculosis found post- mortem in a geriatric population. Z Gerontol, v. 22, p. 3। - -4, 1989

15. MINISTÉRIO DA SAÚDE. Projetos e Programas. Disponível em: http://www.saude.gov.br/programas/Tuberculose/intro.html. Acesso em: 29 set. 2004

16. MARTINEZ, M.J. et al. Estudio multicéntrico de la tuberculosis en autopsias clinicas en Andalucia, en el periodo 1973-88. Rev Clin Esp, v. I88, p. 273-7, 1991.

17. POST, C.; WARTENHORST, H.S. Clinically unrecognized tuberculosis in autopsy material. Dtsch Med Wochenschr, v. I04 n. 13, p. 461-6, 1979.

18. RIQUET, M. et al. Thoracic duct tributaries from intrathoracic organs. Ann Thorac Surg, v. 73, p. 892-8, 2002.

19. ROWINSKA-ZAKRZEWSKA, E. et al. Tuberculosis in the autopsy material: analysis of 1,500 autopsies performed between 1972 and 1991 in the Institute of Tuberculosis and Chest Disease, Warsaw, Poland. Tuber Lung Dis, v. 76 , p. 349-54, 1994 
20. VALLEJO, E. et al. Tuberculosis: diagnóstico postmortem y su relación con el diagnóstico clínico. Rev.Med. Hosp. Gen.Mex, v. 57, n. 4, p.142-5, 1995.

2I.VEIGA, G.M. et al.Tuberculosis miliar: estudio autopsico de 29 casos. An Med Interna, v. 12, p. 17-20, 1995.

22. VENTURA, A.A. Aspectos epidemiológicos da tuberculose na região de saúde de Botucatu-SP, de 1993 a 1998. Dissertação (mestrado) - Faculdade de Medicina de Botucatu, 2001.

23. WORLD HEALTH ORGANIZATION (WHO). Tuberculosis: strategy and operations. Disponível em http://www.who.org. Acesso em: 29 set. 2004. 